\title{
AFECTIVIDAD POSITIVA Y NEGATIVA EN EL FUTURO DOCENTE: RELACIONES CON SU RENDIMIENTO ACADÉMICO, SALUD MENTAL Y SATISFACCIÓN CON LA VIDA
}

\author{
Ruth Pinedo González \\ María José Arroyo González \\ César Caballero San José \\ Universidad de Valladolid
}

\begin{abstract}
RESUMEN: El afecto está formado por dos dimensiones dominantes: el afecto positivo (AP) y el afecto negativo (AN). Ambas dimensiones están relacionadas con el ajuste psicológico de la persona y la satisfacción con la vida. El presente estudio tiene un carácter exploratorio y pretende hacer un primer análisis correlacional entre distintos constructos: disposición emocional, rendimiento académico, salud mental y satisfacción con la vida en futuros docentes. La muestra estaba compuesta por 143 estudiantes de diferentes Grados de Educación, de las especialidades de Primaria e Infantil. Para ellos se han usado las adaptaciones al castellano de los siguientes instrumentos de evaluación: el Positive and Negative Affect Schedule (PANAS), el Mental Health Inventory (MHI-5) y la Satisfaction With Life Scale (SWLS). Entre los resultados de mayor interés se encontró que el afecto positivo se relacionó con el rendimiento académico, la salud mental y la satisfacción con la vida. Además los afectos positivos y negativos junto con la satisfacción con la vida se constituyeron como las variables predictoras de la salud mental del futuro profesorado. Un análisis y discusión más extensa de los resultados se incluye en el documento.
\end{abstract}

PALABRAS CLAVE: Emoción, satisfacción con la vida, salud mental, rendimiento académico, profesorado.

POSITIVE AND NEGATIVE AFFECT IN THE FUTURE TEACHER: RELATIONSHIPS WITH THEIR ACADEMIC ACHIEVEMENT, MENTAL HEALTH AND SATISFACTION WITH LIFE

\footnotetext{
ABSTRACT: Affects are composed of two key dimensions: the positive affect (PA) and negative affect (NA). Both dimensions are related to psychological
} 
adjustment of the person and life satisfaction. This study is exploratory in nature and aims to make a first correlational analysis between different constructs: emotional disposition, academic achievement, mental health and life satisfaction in a sample of 143 student teachers. We have used the following scales adapted to the culture: The Positive and Negative Affect Schedule (PANAS), the Mental Health Inventory (MHI-5) and the Satisfaction With Life Scale (SWLS). Among the most interesting results it was found that positive affect was associated with academic achievement, mental health and life satisfaction. Positive and negative affects and satisfaction with life were formed as predictors of future teachers' mental health. Extensive analysis and discussion of the results is included in the document.

KEYWORDS: Emotion, satisfaction with life, mental health, academic achievement, teacher.

Recibido: 21/03/2016

Aceptado: 18/07/2016

Correspondencia: Ruth Pinedo González, Universidad de Valladolid, Facultad de Educación, Departamento de Psicología, Campus universitario María Zambrano, Plaza de la Universidad, 1, 47005 Segovia. Email: ruth.pinedo@psi.uva.es.

\section{INTRODUCCIÓN}

\section{Disposición emocional}

Las emociones son estados mentales y afectivos que comprenden sentimientos, expresiones corporales, reacciones fisiológicas y tendencias a actuar de una manera específica (Frijda, Manstead y Bem, 2000). Como apunta Vázquez (2009), son aquellas experiencias anímicas y emocionales, que nos informan sobre la marcha de nuestra vida. En la investigación sobre bienestar, el afecto ha sido uno de los constructos pioneros en ser analizado. Los trabajos realizados en los últimos años concluyen que el afecto está formado por dos dimensiones dominantes: afecto positivo y afecto negativo (Lopez-Gomez, Hervas y Vazquez, 2015). Así, el afecto positivo (AP) abarca la dimensión de emocionalidad positiva, energía, afiliación y domino de un individuo. Las personas con bajo AP manifiestan desinterés y aburrimiento, por el contrario, aquellos sujetos con alto AP experimentan con frecuencia sentimientos de satisfacción, gusto, entusiasmo, energía, amistad, unión, afirmación y confianza. En cuanto al afecto negativo (AN) se refiere a la dimensión de la sensibilidad temperamental de un individuo ante estímulos negativos. Altos índices de AN se han relacionado con emociones negativas como miedo o ansiedad, tristeza o depresión, culpa, hostilidad e insatisfacción, actitudes negativas y pesimismo, problemas o quejas somáticas, así como con insatisfacción y depreciación negativa de uno mismo y de los demás (Lopez-Gomez et al., 2015; Watson y Clark, 1984). El AP y AN son dimensiones altamente diferenciadas e independientes como apuntan distintas investigaciones, aun- 
que a priori pudiesen parecer opuestos, es decir, elementos extremos de una misma dimensión. Por tanto, el afecto negativo incluye emociones y estados de ánimo con contenido subjetivo desagradable (ira, tristeza, ansiedad, preocupación, celos). Las personas con alto afecto negativo experimentan desinterés, aburrimiento, aflicción, culpa, nerviosismo, angustia, miedo, vergüenza y envidia. Constituye un factor de riesgo de enfermedades. El afecto positivo incluye estados de ánimo y emociones con contenido subjetivo agradable (alegría, cariño, gozo, interés por las cosas). Las personas con alto afecto positivo experimentan sentimientos de satisfacción, entusiasmo, energía y confianza. Se relaciona con extroversión, optimismo y resiliencia.

\section{Disposición emocional en el docente}

La enseñanza ha sido considerada una de las profesiones con mayor riesgo laboral por estrés según el proyecto Stress Impact (Zijlstra, 2006) realizado durante cuatro años en diferentes países de Europa (2002-2006). El informe pone de manifiesto cómo las causas de este malestar docente son muchas veces más subjetivas que objetivas, ya que es una valoración que la persona hace de su entorno, es decir, de cómo vive los acontecimientos que le rodean, no tanto de la situación en sí. Así, es necesario que el docente se conozca, sepa lo que vive, lo que siente, espera o quiere, que comprenda bien los límites y las posibilidades de sus relaciones con los otros, siendo dueño de sus emociones. Este conocimiento emocional le será de utilidad para sus alumnos, ya que el maestro es un modelo para ellos: él será quien les ayude a conocerse, a analizar lo que les pasa, a ser felices y a disfrutar de la vida. Los docentes son modelos de comportamiento para sus alumnos en dos niveles: cognitivo y emocional. Por tanto, esta capacidad socioafectiva es relevante para los futuros maestros desde una doble perspectiva: para sí mismo y para los demás, aunque existen pocas investigaciones relativas a la disposición emocional de los docentes (Pertegal-Felices, Castejón-Costa y Martínez, 2011).

\section{Relaciones entre afectividad y salud mental}

Numerosos trabajos se han centrado en analizar la relación entre la afectividad y la salud mental, en concreto, en la relación entre los afectos positivos y negativos con los problemas psicológicos. Así, encontramos que la ansiedad se asocia con un estado afectivo negativo, sin apenas relación con el afecto positivo. Por otra parte, la depresión se caracteriza por un estado mixto de alto afecto negativo y bajo afecto positivo (Watson et al., 1988). Resultan relevantes otras correlaciones encontradas entre el afecto y diversas dimensiones de la personalidad, por ejemplo, vemos cómo el afecto positivo se asocia con extroversión, mientras que el afecto negativo tiene una alta correlación con neuroticismo (Watson et al., 1988). Avia y Sánchez (1995) afirman que los diferentes niveles de afecto positivo y negativo que vivencia un sujeto, permiten clasificar a las personas en función del grado en el que experimentan o no determinadas emociones.

En el trabajo realizado por Esteras, Chorot y Sandín (2014) sobre predicción del burnout y el estrés en los docentes encontraron que la afectividad negativa actuaba como factor general de vulnerabilidad. Así, la baja satisfacción laboral y el estrés laboral fueron poderosos predictores de la baja disposición emocional negativa. 


\section{Relaciones entre afectividad y satisfacción con la vida}

Diener y Tay (2015) definen la satisfacción con la vida como una evaluación global que la persona hace sobre su vida. Así, la persona somete a análisis los aspectos tangibles de su vida, sopesa lo bueno frente a lo malo y compara con un criterio que ella misma elige, Ilegando a un juicio final sobre su vida. Por ello, cuando se inicia un estudio sobre la satisfacción con la vida se debe centrar en los juicios subjetivos que hace la persona sobre su vida, a través de una evaluación global que hace sobre la misma (Atienza, Pons, Balaguer y García-Merita, 2000; Lucas, Diener y Larsen, 2003).

La afectividad positiva es uno de los aspectos que forman parte de la felicidad o satisfacción con la vida, como lo refrenda el estudio realizado por Lyubomirsky, King y Diener (2005). Estos autores realizaron un estudio donde encontraron que el AP puede ser la causa de algunas de las características, recursos y éxitos deseables que correlacionan con la felicidad. El AP incluye cualidades como la confianza, el optimismo, la autoeficacia, la sociabilidad, la actividad, la energía, la conducta prosocial, el bienestar físico, el afrontamiento efectivo, la originalidad y la flexibilidad. Diferentes estudios (Diener, Suh, Lucas y Smith, 1999; Vera, Rodríguez y Grubits, 2009) han encontraron correlaciones significativas entre satisfacción con la vida y afecto positivo y las relaciones entre éste y autopercepciones más positivas.

El modelo conceptual de Lyubomirsky et al. (2005) propone que la felicidad surge porque el AP engendra éxito, lleva a la persona a pensar, sentir y actuar en la línea de la construcción de recursos y la consecución de objetivos (Elliot y Thrash, 2002; Lyubomirsky, 2001). En esta misma línea está la propuesta de Fredrickson (2000), en su "Teoría de la Construcción y la Ampliación", construyendo así recursos personales más duraderos y amortiguando las emociones negativas.

Por otro lado, en la versión portuguesa de la Escala de Satisfacción con la Vida (SWLS) realizada por Queirós, Fernández-Berrocal, Extremera, Carral y Queirós (2005) se encontró que a mayores niveles de disposición emocional positiva se dan mayores niveles de satisfacción con la vida. De este modo, las personas que comprenden bien cómo se sienten en cada momento, poseen una mayor capacidad para prolongar sus estados positivos e interrumpir los estados emocionales negativos, en consecuencia obtienen un mayor bienestar psicológico y una mayor satisfacción con la vida que aquellas personas que no comprenden ni regulan sus emociones. En Landa, Lopez-Zafra, de Antonana y Pulido (2006) también se mostró una correlación entre la SWLS y dos dimensiones de la Inteligencia Emocional en una muestra universitaria. Por su parte, en estudios españoles también se muestran relaciones de la disposición emocional con la satisfacción con la vida (Extremera, Durán y Rey, 2007).

Resulta imprescindible un breve apunte sobre el bienestar subjetivo, ya que es un constructo que maneja la relación entre la disposición emocional y la satisfacción con la vida. Este concepto ha sido muy importante en la investigación psicológica en años precedentes, caracterizadas por la complejidad debido a las diferentes perspectivas y teorías explicativas del mismo (Lucas et al., 2003). En este constructo se han identificado dos componentes diferenciados: el primero emocional, relacionado con los aspectos positivos y un segundo componente cognitivo, al cual se le ha denominado satisfacción con la vida (Diener et al., 1999). Si bien las reacciones afectivas son a menudo respuestas a factores inmediatos y de duración corta, las posiciones de sa- 
tisfacción vital pueden reflejar una perspectiva a largo plazo. Podemos concluir que existe un cierto grado de convergencia entre la disposición emocional y satisfacción con la vida ya que ambos componentes dependen de una apreciación evaluativa (Pavot y Diener, 2009; Vera, Freire, López y García, 2010).

\section{Relaciones entre afectividad y rendimiento académico}

Se ha señalado también la relación entre una baja disposición emocional con bajos niveles de bienestar y ajuste psicológico del alumnado, con una menor cantidad y calidad en las interacciones personales, así como un descenso en el rendimiento académico (Extremera et al., 2007; Extremera y Fernández-Berrocal, 2004). El estudio muestra evidencias de la relación entre altas puntuaciones en disposición emocional con mayores niveles de eficacia académica, así como un mayor nivel de vigor, dedicación y eficacia (engagement).

\section{Afectividad, rendimiento académico, satisfacción con la vida y salud mental}

En resumen, la disposición emocional tiene una gran influencia en distintos ámbitos de la vida tales como: la prevención de conductas de riesgo (Brackett y Mayer, 2003), las relaciones familiares (Chang, 2005), la competencia social (Brasseur y Gregoire, 2011), el ajuste socioescolar del alumnado (Pena y Repetto, 2008) y de especial interés para el trabajo que aquí presentamos, la relación con el bienestar subjetivo (Bar-On, 2005), el bienestar personal y laboral (Pena, Rey y Extremera, 2012), la salud mental (Bhullar, Schutte y Malouff, 2013) y la felicidad (Bar-On, 2010). En el ámbito educativo, la disposición emocional desempeñaría un papel importante en procesos de estrés y burnout (Extremera et al., 2007). Así, las personas con una disposición emocional positiva comprenden las emociones originadas en su entorno, analizan sus causas y consecuencias y saben regular estas emociones con estrategias ajustadas (Salovey, Mayer y Caruso, 2002). Otros estudios apuntan que estas habilidades ayudan a una mejor percepción de autoeficacia y un mejor afrontamiento de los conflictos acaecidos en el entorno laboral (Bar-On, Brown, Kirkcaldy y Thome, 2000; Durán, Extremera y Rey, 2004; Limonero, Tomás Sábado, Fernández-Castro y Gómez-Benito, 2004; Nikolaou y Tsaousis, 2002).

Encontramos estudios que relacionan los afectos positivos y negativos, con el ajuste psicológico de la persona y su desempeño académico (Arguís, Bolsas, Hernández y Salvador, 2012; Beckett Vermehren, Zubiri Gonzalez y Schultzendorff Hoyl, 2015; Vera et al., 2009) si bien no existen estudios previos que evalúen los niveles de afectividad negativa y positiva experimentada por los estudiantes del Grado/Máster de Educación o futuros docentes en España siendo un aspecto importante a estudiar porque está muy relacionado con el ajuste psicológico de la persona y en definitiva, con la calidad docente.

A la luz de todo lo expuesto anteriormente este estudio se plantea los siguientes objetivos: (1) evaluar el nivel de afectividad positiva y negativa experimentada por futuros docentes y (2) explorar las relaciones existentes entre la afectividad positiva y negativa con los niveles de rendimiento académico, salud mental y satisfacción con la vida en futuros docentes. 


\section{Método}

\section{Participantes}

La muestra está formada por 143 personas estudiantes de alguno de los Grados de Educación de las diferentes provincias de Castilla y León. La muestra fue seleccionada mediante un muestreo no probabilístico de tipo casual o accidental, es decir, se seleccionó como muestra participante a todo el alumnado al que se tenía acceso directo. La edad media de los participantes es de 21,16 años (d.t. = 5,02), con un mínimo de 18 años y un máximo de 54 años. El 81,1\% de la muestra son mujeres y el $60,1 \%$ se encuentran cursando $1^{\circ}$ curso del Grado. La muestra analizada se encuentra matriculada en el Grado de Educación Infantil (41.3\%), el Grado de Educación Primaria (45.5\%) y el Máster de Educación Secundaria (13.3\%).

\section{Instrumentos}

La toma de datos se realizó a partir de los resultados de tres pruebas estandarizadas y de uso frecuente como son el Positive and Negative Affect Schedule (PANAS; Watson, Clark y Tellegen, 1988), el Mental Health Inventory (MHI-5; Berwick et al., 1991) y la Satisfaction With Life Scale (SWLS; Diener, Emmons, Larsen y Griffin, 1985). Como complemento a los datos anteriores, se realizó un cuestionario a todos los participantes.

Afecto positivo y afecto negativo. Se utilizó la versión adaptada al castellano del cuestionario de afecto positivo y negativo PANAS (López-Gómez et al., 2015). El cuestionario se compone de 20 items, diez de los cuales hacen referencia a la subescala de afecto positivo (AP) y 10 a la subescala de afecto negativo (AN). Los ítems de la escala son de tipo Likert de 5 puntos ( $1=$ Muy poco o Nada a $5=$ Extremadamente). La adaptación al castellano presenta propiedades psicométricas adecuadas equivalentes a la de la escala original. La subescala de afecto negativo incluye emociones y estados de ánimo con contenido subjetivo desagradable (ira, tristeza, ansiedad, preocupación, celos). Mientras que la subescala de afecto negativo incluye estados de ánimo y emociones con contenido subjetivo agradable (alegría, cariño, gozo, interés por las cosas) (Beckett Vermehren et al., 2015).

Salud mental. El MHI-5 es una de las ocho dimensiones, denominada salud mental, del cuestionario Short Form 36 Health Survey (SF-36; Ware, Kosinski y Keller, 1994). El inventario se compone de cinco items que miden salud mental y se responden en una escala Likert de 6 puntos $(1=$ Nunca a $6=$ Siempre). En este estudio se ha utilizado la adaptación al castellano que presenta propiedades psicométricas adecuadas equivalentes a la escala original (Vilagut et al., 2005).

Satisfacción con la vida. Finalmente se utilizó la versión adaptada al castellano de la escala de Satisfacción con la Vida (SWLS) de Diener et al. (1985) realizada por Garrido, Fernández, Villalba, Pérez y Fernández (2010) con población universitaria. Consta de cinco ítems tipo Likert con cinco puntos (1=Totalmente en desacuerdo a $5=$ Totalmente de acuerdo). La adaptación al castellano presenta propiedades psicométricas adecuadas equivalentes a la escala original.

Como se ha indicado, los datos anteriores se completaron con una entrevista estructurada que contenía preguntas relativas a variables sociodemográficas y variables académicas (ver Tabla 1). 
Tabla 1. Instrumento de evaluación del estudio (Variables-Items-Preguntas y Escalas de respuesta utilizadas)

\begin{tabular}{|c|c|c|c|}
\hline Variables & Ítems & Pregunta & Escala de respuesta \\
\hline \multirow{4}{*}{ Académicas } & Curso & ¿En qué curso estás matriculado? & $\begin{array}{ll}\text { 1. } & 1^{\circ} \\
\text { 2. } & 2^{\circ} \\
\text { 3. } & 3^{\circ} \\
\text { 4. } & 4^{\circ} \\
\text { 5. } & \text { Máster } \\
\end{array}$ \\
\hline & Especialidad & ¿Qué especialidad estás cursando? & $\begin{array}{ll}\text { 1. } & \text { Infantil } \\
\text { 2. } & \text { Primaria } \\
\text { 3. } & \text { Titulación } \\
\text { Conjunta } \\
\text { 4. } & \text { Máster Secundaria } \\
\end{array}$ \\
\hline & $\begin{array}{l}\text { Rendimiento } \\
\text { académico }\end{array}$ & $\begin{array}{l}\text { ¿Cuál ha sido tu nota media en el } \\
\text { curso anterior? }\end{array}$ & \\
\hline & $\begin{array}{c}\text { Fracaso } \\
\text { académico }\end{array}$ & $\begin{array}{l}\text { ¿Qué número de asignaturas has } \\
\text { tenido que repetir la matrícula? }\end{array}$ & \\
\hline $\begin{array}{l}\text { Afectividad } \\
\text { Positiva y } \\
\text { Negativa }\end{array}$ & & $\begin{array}{l}\text { Motivado / Molesto / Emocionado / De } \\
\text { malas / Firme / Culpable / Temeroso / } \\
\text { Agresivo / Entusiasmado / Orgulloso } \\
\text { / Irritable / Alerta / Avergonzado / } \\
\text { Inspirado / Nervioso / Decidido / } \\
\text { Atento / Inquieto / Activo / Seguro } \\
\end{array}$ & $\begin{array}{ll}\text { 1. } & \text { Muy poco o Nada } \\
\text { 2. } & \text { Algo } \\
\text { 3. } & \text { Intermedio } \\
\text { 4. } & \text { Bastante } \\
\text { 5. } & \text { Mucho } \\
\end{array}$ \\
\hline $\begin{array}{l}\text { Satisfacción } \\
\text { con la vida }\end{array}$ & & $\begin{array}{l}\text { 1. La mayoría de los aspectos de mi } \\
\text { vida son como quiero que sean } \\
\text { 2. Hasta ahora he conseguido de } \\
\text { la vida las cosas que considero } \\
\text { importantes } \\
\text { 3. Estoy satisfecho/a con mi vida } \\
\text { 4. Si pudiera vivir mi vida otra vez, } \\
\text { la repetiría tal y como ha sido } \\
\text { 5. Las circunstancias de mi vida son } \\
\text { buenas }\end{array}$ & $\begin{array}{ll}\text { 1. } & \text { Muy en } \\
\text { desacuerdo } \\
\text { 2. } & \text { Desacuerdo } \\
\text { 3. } & \text { Intermedio } \\
\text { 4. } & \text { De acuerdo } \\
\text { 5. } & \text { Muy de acuerdo }\end{array}$ \\
\hline Salud Mental & & $\begin{array}{l}\text { 1. Durante las } 4 \text { últimas semanas, } \\
\text { ¿Cuánto tiempo estuvo muy } \\
\text { nervioso? } \\
\text { 2. Durante las } 4 \text { últimas semanas, } \\
\text { ¿Cuánto tiempo se sintió calmado } \\
\text { y tranquilo? } \\
\text { 3. Durante las } 4 \text { últimas semanas, } \\
\text { ¿Cuánto tiempo se sintió } \\
\text { desanimado y triste? } \\
\text { 4. Durante las } 4 \text { últimas semanas, } \\
\text { ¿Cuánto tiempo se sintió feliz? } \\
\text { 5. Durante las } 4 \text { últimas semanas, } \\
\text { ¿Cuánto tiempo se sintió tan } \\
\text { bajo de moral que nada podía } \\
\text { animarle? }\end{array}$ & $\begin{array}{l}\text { 1. Nunca } \\
\text { 2. Rara vez } \\
\text { 3. Algunas veces } \\
\text { 4. } \text { Bastante } \\
\text { 5. } \\
\text { 6. } \\
\text { Siempre }\end{array}$ \\
\hline
\end{tabular}




\section{Procedimiento}

Las preguntas de la entrevista ad hoc elaborada para este estudio junto con los items de las escalas mencionadas anteriormente se incluyeron en un formulario on-line a través de la herramienta gratuita Google Formularios. Dado que todos los participantes en este estudio eran mayores de edad se solicitó su consentimiento para enviarles por correo electrónico el enlace en el que podían contestar el cuestionario de forma anónima. El enlace se envió a través del campus virtual de la Universidad de Valladolid, con la colaboración de un grupo de profesores para captar alumnado de diferentes grados y cursos. Al inicio del cuestionario on-line se proporcionaba información detallada sobre la finalidad investigadora del estudio y el tratamiento de los datos. En todo momento, se preservó el anonimato y confidencialidad de las personas participantes del estudio ya que no se solicitaron datos personales ni códigos de identificación.

\section{Análisis de datos}

La contrastación de todas las hipótesis así como sus cálculos correspondientes se Ilevaron a cabo con el programa informático SPSS v.20. Se Ilevó a cabo un análisis de varianza (ANOVA) de un factor para analizar las diferencias entre los grupos, correlaciones de Pearson para analizar las relaciones entre las variables y un análisis de regresión múltiple bajo el método paso a paso para evaluar las posibles variables asociadas con el rendimiento y fracaso académico en futuros docentes.

\section{Resultados}

En primer lugar, este estudio se planteó evaluar el nivel de afectividad positiva y negativa experimentada por futuros docentes matriculados en diferentes grados académicos. Tal y como podemos ver en la Tabla 2 los niveles de afectividad positiva son mayores que los niveles de afectividad negativa. Al comparar, mediante el ANOVA de un factor, si existían diferencias en los niveles de afectividad positiva y negativa en el alumnado matriculado en infantil, primaria o secundaria se encontró que sí que existían diferencias significativas $\left(\mathrm{F}_{(2,140)}=6.03, \mathrm{p}=.003\right)$ en la subescala de afectividad positiva, pero no existían en la subescala de afectividad negativa $\left(\mathrm{F}_{(2,140)}=.09, \mathrm{p}=.91\right)$. En concreto, estas diferencias se dieron entre el alumnado matriculado en educación infantil y el alumnado matriculado en educación primaria $(p=.002)$ de manera que el alumnado matriculado en infantil mostraba mayor nivel de afectividad positiva que el de primaria.

También se midieron los niveles de salud mental y satisfacción con la vida en la muestra (ver Tabla 2) pero no se encontraron diferencias significativas entre los diferentes grupos de alumnado ni en salud mental $\left(F_{(2,140)}=1.74, p=.18\right)$ ni en satisfacción con la vida $\left(\mathrm{F}_{(2,140)}=1.36, \mathrm{p}=.26\right)$.

En segundo lugar, este estudio se planteó explorar las relaciones existentes entre la afectividad positiva y negativa con los niveles de rendimiento académico, salud mental y satisfacción con la vida en futuros docentes. Para ello se realizó en un primer momento un análisis de correlación bivariada de Pearson (ver Tabla 3). 
Tabla 2. Estadísticos descriptivos de la variable afectividad positiva y negativa en función de la especialidad del Grado cursado

\begin{tabular}{ccccc}
\cline { 2 - 5 } & Muestra general & $\begin{array}{c}\text { Alumnado de } \\
\text { Educación } \\
\text { Infantil }\end{array}$ & $\begin{array}{c}\text { Alumnado de } \\
\text { Educación } \\
\text { Primaria }\end{array}$ & $\begin{array}{c}\text { Alumnado de } \\
\text { Educación } \\
\text { Secundaria }\end{array}$ \\
\cline { 2 - 5 } & Media (d.t.) & Media (d.t.) & Media (d.t.) & Media (d.t.) \\
\hline Afecto Positivo & $33.48(5.26)$ & $35.05(4.91)$ & $31.90(5.41)$ & $34.00(4.34)$ \\
\hline $\begin{array}{c}\text { Afecto } \\
\text { Negativo }\end{array}$ & $19.5(6.63)$ & $19.29(6.88)$ & $19.75(6.98)$ & $19.21(4.49)$ \\
\hline Salud Mental & $20.97(4.69)$ & $21.85(4.72)$ & $20.34(4.90)$ & $20.47(3.50)$ \\
\hline $\begin{array}{c}\text { Satisfacción } \\
\text { con la vida }\end{array}$ & $18.31(3.83)$ & $18.91(3.90)$ & $17.78(3.77)$ & $18.26(3.82)$ \\
\hline
\end{tabular}

En cuanto a las variables académicas, se encontraron correlaciones significativas entre las variables rendimiento académico y afecto positivo, de manera que mayores niveles de afecto positivo se relacionaban con un mejor rendimiento académico. En cuando a la variable denominada fracaso académico se correlacionó de forma negativa con la salud mental, de manera que un mayor fracaso académico se correlacionó con un peor estado de salud mental.

El afecto positivo se correlacionó de forma positiva y fuerte con la salud mental y la satisfacción con la vida, de manera que mayores niveles de afecto positivo se relacionaron con mejor salud mental y mayor satisfacción con la vida.

El afecto negativo se correlacionó de forma negativa y fuerte con la salud mental y la satisfacción con la vida, de manera que mayores niveles de afecto negativo se relacionaron con un peor estado de salud mental y menor satisfacción con la vida.

Finalmente, salud mental y satisfacción con la vida mostraron una alta correlación positiva, de manera que mayores niveles de satisfacción con la vida se relacionaban con mayores niveles de salud mental.

Tabla 3. Correlaciones bivariadas entre las variables del estudio

\begin{tabular}{rcccccc}
\hline & $\begin{array}{c}\text { Rendimiento } \\
\text { académico }\end{array}$ & $\begin{array}{c}\text { Fracaso } \\
\text { académico }\end{array}$ & $\begin{array}{c}\text { Afecto } \\
\text { positivo }\end{array}$ & $\begin{array}{c}\text { Afecto } \\
\text { negativo }\end{array}$ & $\begin{array}{c}\text { Salud } \\
\text { mental }\end{array}$ & $\begin{array}{c}\text { Satisfacción } \\
\text { con la vida }\end{array}$ \\
\hline $\begin{array}{r}\text { Rendimiento } \\
\text { académico }\end{array}$ & $\mathbf{1}$ & & & & & \\
$\begin{array}{r}\text { Fracaso } \\
\text { académico }\end{array}$ & -.03 & $\mathbf{1}$ & & & & \\
Afecto positivo &. $\mathbf{2 2 *}$ & -.07 & $\mathbf{1}$ & & & \\
Afecto negativo & .09 & .12 & .005 & $\mathbf{1}$ & & \\
$\begin{array}{r}\text { Salud mental } \\
\text { Satisfacción con } \\
\text { la vida }\end{array}$ & .08 & $-.021^{*}$ & $\mathbf{. 4 1 * *}$ & $\mathbf{- . 6 1 * *}$ & $\mathbf{1}$ & \\
\hline
\end{tabular}

*La correlación es significativa al nivel .05 (bilateral)

**La correlación es significativa al nivel .001 (bilateral) 
Como último paso en el análisis de datos, se analizó la relación entre las variables con el objetivo de plantear un modelo predictivo de la variable salud mental a partir de las variables afectividad positiva y negativa, satisfacción con la vida y variables académicas.

En relación a la salud mental, en el análisis de regresión lineal múltiple entraron tres variables y la relación entre estas viene especificada en la siguiente ecuación:

Salud Mental $=11.36-.36($ Afectividad negativa $)+.27$ (Afectividad Positiva $)+.41$ (Satisfacción con la vida)

El afecto negativo entra en primer paso en sentido inverso $\left(\mathrm{F}_{(1,137)}=81, \mathrm{p}=.001\right)$, el afecto positivo entra en segundo paso en sentido directo $\left(F_{(2,136)}=77.65, p=.001\right)$ y la satisfacción con la vida entra en tercer paso en sentido directo $\left(F_{(3,136)}=73.98\right.$, $\mathrm{p}=.001)$. Las tres variables explican un $62.2 \%$ de la varianza de la variable salud mental (ver Tabla 4).

Tabla 4. Coeficientes tipificados y sus valores de probabilidad del análisis de regresión múltiple para la variable Salud Mental

\begin{tabular}{|c|c|c|c|c|c|c|}
\hline \multirow{2}{*}{ Modelo } & & \multicolumn{2}{|c|}{$\begin{array}{l}\text { Coeficientes no } \\
\text { estandarizados }\end{array}$} & \multirow{2}{*}{$\begin{array}{c}\text { Coeficientes } \\
\text { tipificados } \\
\text { Beta } \\
\end{array}$} & \multirow[b]{2}{*}{$\mathrm{t}$} & \multirow[b]{2}{*}{ Sig. } \\
\hline & & B & $\begin{array}{c}\text { Error } \\
\text { típ. }\end{array}$ & & & \\
\hline & Constante & 11,357 & 2,026 & & 5,606 & ,000 \\
\hline & Afectividad Negativa &,- 360 & 038 &,- 527 & $-9,536$ & ,000 \\
\hline & Afectividad Positiva & ,273 &, 047 &, 317 & 5,758 &, 000 \\
\hline & Satisfacción con la vida & 410 &, 073 & ,322 & 5,627 &, 000 \\
\hline
\end{tabular}

a. Variable dependiente: Salud Mental

En resumen, el análisis de regresión múltiple llevado a cabo mostró la existencia de una relación significativa entre las variables de manera que se puede estimar la salud mental de los futuros docentes a través de las variables afectividad positiva, afectividad negativa y satisfacción con la vida.

\section{DisCuSión Y CONCLUSIONES}

El presente estudio tiene un carácter exploratorio y pretende hacer un primer análisis correlacional entre distintos constructos: disposición emocional, rendimiento académico, salud mental y satisfacción con la vida. Es un tema de interés, ya que no existen estudios anteriores que evalúen los niveles de afectividad negativa y positiva experimentada por los estudiantes del grado de educación o futuros docentes en España y es un aspecto importante a estudiar porque está muy relacionado con el ajuste psicológico de la persona y la calidad docente, más aún cuando la labor docente ha sido clasificada como una de las profesiones que sufre mayor estrés (Zijlstra, 2006). Es importante identificar las relaciones existentes entre 
los constructos definidos para plantear posibles intervenciones futuras para mejorar la disposición emocional del profesorado, su calidad docente y su satisfacción con la vida.

Mediante los resultados obtenidos en este trabajo se puede concluir que el colectivo de futuros docentes presenta en general mayores niveles de afectividad positiva que negativa. Pero al analizar los niveles de afectividad según los diferentes grupos docentes se han encontrado diferencias significativas, ya que el alumnado de Educación Infantil presenta mayores niveles de afectividad positiva que el alumnado de Educación Primaria. En afectividad negativa no se han encontrado diferencias entre los diferentes grupos.

Al explorar las relaciones entre las variables estudiadas se ha encontrado que las variables relacionadas con la disposición emocional del alumnado de educación se relacionan de forma significativa y elevada con las variables de salud mental y satisfacción con la vida. En concreto, el afecto positivo se correlacionó de forma directa con la salud mental y la satisfacción con la vida, mientras que el afecto negativo lo hizo igualmente pero de forma inversa. Estos hallazgos se encuentran en la línea de otras investigaciones similares que han encontrado relación fuerte y significativa entre estos constructos (Bastian, Kuppens, De Roover y Diener, 2014; Esteras et al., 2014; Lyubomirsky et al., 2005). En concreto, Esteras et al. (2014) señalaron que altas puntuaciones en afecto negativo se convertían en factores de vulnerabilidad en el profesorado. Por tanto, sería deseable realizar intervenciones en el profesorado que les ayuden a controlar y gestionar sus emociones, ya que actuarán como protectores del estrés, actuarán como protectores del síndrome de burnout y le ayudarán en el desarrollo diario de su trabajo. La disposición emocional positiva también correlaciona con una alta satisfacción con la vida y con la salud mental. El afecto positivo incluye cualidades como la confianza, el optimismo, la autoeficacia, la sociabilidad, la actividad, la energía, la conducta prosocial, el bienestar físico, el afrontamiento efectivo, la originalidad y la flexibilidad. En la misma línea Elliot y Thrash (2002) plantean que los afectos positivos engendrarán éxitos y felicidad y poco a poco hace que los sujetos construyan recursos personales más duraderos y aprendan a amortiguar las emociones negativas (Lyubomirsky, King y Diener, 2005).

También se encontró una relación significativa y directa entre el afecto positivo y el rendimiento académico siguiendo la misma línea que investigaciones anteriores (Extremera et al., 2007; González, Danolo, Rinaudo y Paoloni, 2011) donde se relacionó con mayor nivel de vigor y engagement. Variables como el fracaso académico se relacionó de forma única e inversa con la salud mental, pero no se relacionó de forma significativa con ninguna de las variables relacionadas con la afectividad del alumnado. Finalmente, también se encontró una fuerte relación significativa y directa entre la salud mental y la satisfacción con la vida, tal y como se han encontrado en otras investigaciones anteriores (Diener y Tay, 2015).

Nuestro interés en este estudio es identificar las relaciones existentes entre los constructos definidos para diseñar programas de intervención en el profesorado. En base a los resultados encontrados, mejorar los niveles de afectividad positiva y disminuir los niveles de afectividad negativa en los maestros ayudará a mejorar su salud mental y por consiguiente, su ajuste psicológico. Docentes con mejor salud 
mental serán docentes más sanos y con mejor disposición para afrontar situaciones potencialmente estresantes relacionadas con su desempeño laboral.

La investigación que aquí nos ocupa tiene una gran relevancia en la práctica y en la formación de los futuros docentes: ¿qué se supone que tiene que aprender un futuro docente? Mucho se ha debatido en torno a esta pregunta. La respuesta a ésta pregunta viene marcada desde el modelo europeo de formación por el concepto de competencia. La legislación educativa actual y las propuestas de las distintas universidades sobre formación de futuros maestros engloban el concepto de competencia en cinco ámbitos: saber, saber ser, saber hacer qué, saber hacer cómo y saber estar (OECD, 2006). El tema que aquí nos ocupa, las emociones y los afectos, estarían englobados en la competencia del saber ser y en particular en la competencia intra e interpersonal, que engloba el conjunto de características y actitudes personales hacia uno mismo, hacia los demás y hacia la propia profesión. La educación emocional es una parte fundamental de la formación integral de la personalidad y por ello debería estar presente en la preparación de todos los profesionales que trabajan con personas y ocupar un papel destacado en la formación de los maestros. La realidad dista mucho de esta idea y vemos que está prácticamente ausente de los actuales planes de estudio y en los documentos de referencia tales como: Espacio Europeo de Educación Superior, la Conferencia de Decanos y Directores en Magisterio y Educación, el Libro Blanco del Título de Grado en Magisterio (ANECA, 2005) y el propio Boletín Oficial del Estado han optado por el discurso técnico de la formación profesional (Palomero y Torrego, 2004), dejando muy poco espacio para las competencias socioafectivas. Urge por tanto, retomar la reflexión sobre las competencias personales y profesionales en los futuros maestros planteando modelos de formación inicial y permanente que les ayuden a un desarrollo personal y profesional óptimo.

A la luz de los resultados obtenidos se considera de gran interés realizar intervenciones relacionadas con la gestión y control de las emociones en los futuros docentes, así como en aquellos que ya ejercen. De manera más urgente esta intervención debería realizarse en sectores donde las condiciones labores y los problemas psicosociales sean más negativas. El control emocional les ayudará a comprender bien sus sentimientos en cada momento, prolongar las emociones positivas y minimizar las negativas. Este conocimiento de las emociones les será de utilidad en el trabajo con sus alumnos: enseñarles a controlar y regular sus sentimientos. El objetivo final de este trabajo es obtener información útil para plantear posibles actuaciones e investigaciones futuras que mejoren el ajuste psicológico del profesorado, su calidad docente y su satisfacción con la vida. Entendemos, que la Psicología Positiva puede postularse como una corriente psicológica de utilidad a la hora de perfilar dichas intervenciones. Como apuntó Peterson, Park y Seligman (2005) el principal objetivo de la Psicología Positiva es el estudio del funcionamiento óptimo del ser humano. Su tesis central defiende que las personas pueden aprender a gestionar sus emociones positivas y negativas, practicar nuevas formas más efectivas de afrontamiento, como la resiliencia y la autenticidad en las relaciones sociales, la esperanza, la autoeficacia o la autodeterminación (Salanova, 2011). En definitiva, como bien apuntaba (Fredrickson, 2000) las emociones positivas ayudan a construir recursos personales que conllevan una mayor satisfacción personal y bienestar. 
Los resultados que aquí se presentan responden a una primera fase de una investigación más amplia relacionada con el bienestar emocional de los estudiantes de educación. Este estudio presenta como desventaja principal su diseño de investigación, ya que únicamente nos permite describir la situación del colectivo en relación a las variables estudiadas y analizar las relaciones entre éstas. También contamos con una muestra que no es representativa del alumnado de educación ya que no tiene el tamaño adecuado ni ha sido obtenida a través de métodos de muestreo aleatorios. Aún así, creemos que nos permite formular algunas conclusiones interesantes y establecer propuestas futuras de investigación como son las siguientes:

- ¿Por qué el alumnado de infantil presenta mayores niveles de afectividad positiva? ¿Qué variables están influyendo en el nivel de afectos positivos? ¿Motivación, vocación, compromiso, etc.?

- ¿Por qué la afectividad positiva influye en el rendimiento y el compromiso académico de los futuros docentes?

- ¿Los niveles de afectividad positiva y negativa influyen en los niveles de estrés docente?

- ¿El profesorado con mayores niveles de afectividad positiva afronta mejor y se recupera mejor del estrés que parece acompañar a la labor docente?

\section{ReFERENCIAS BIBLIOGRÁFICAS}

ANECA (2005). Libro blanco título de Grado en Magisterio. Recuperado de: https:// www.uv.es/graus/OPE/ANECA/Ilibres_blancs/libroblanco_magisterio2.pdf.

Arguís, R., Bolsas, A. P., Hernández, S. y Salvador, M. (2012). Programa "Aulas felices". Psicología positiva aplicada a la educación. Recuperado de: http://educaposit. blogspot.com.es/.

Atienza, F. L., Pons, D., Balaguer, I. y García-Merita, M. L. (2000). Propiedades psicométricas de la Escala de Satisfacción con la Vida en adolescentes. Psicothema, 12(2), 331-336.

Avia, M. D. y Sánchez, M. L. (1995). Personalidad: Aspectos cognitivos y sociales. Madrid: Pirámide.

Bar-On, F., Brown, J. M., Kirkcaldy, B. y Thome, E. (2000). Emotional expression and implications for occupational stress; an application of the Emotional Quotient Inventory (EQ-I). Personality and Individual Differences, 28, 1107-1118.

Bar-On, R. (2005). The impact of emotional intelligence on subjetive well-being. Perspectives in Education, 23(23), 41-61.

Bar-On, R. (2010). Emotional intelligence: an integral part of positive psychology. South African Journal of Psychology, 40(11), 54-62.

Bastian, B., Kuppens, P., De Roover, K. y Diener, E. (2014). Is valuing positive emotion associated with life satisfaction? Emotion, 14(4), 639-645. DOI: http://doi. org/10.1037/a0036466.

Beckett Vermehren, E., Zubiri Gonzalez, F. y Schultzendorff Hoyl, A. von (2015). Diseño e implementación de una intervención educativa para elevar el bienestar docente basado en las evidencias de la psicología positiva y las neurociencias. 
Pensamiento Educativo: Revista de Investigación Educacional Latinoamericana, 52(2), 151-171. DOI: http://doi.org/10.7764/PEL.52.2.2015.9.

Berwick, D. M., Murphy, J. M., Goldman, P. A., Ware, J. E., Barsky, A. J. y Weinstein, M. C. (1991). Performance of a five-item mental health screening test. Medical Care, 29, 169-176.

Bhullar, N., Schutte, N. S. y Malouff, J. M. (2013). The Nature of Well-Being: The Roles of Hedonic and Eudaimonic Processes and Trait Emotional Intelligence. The Journal of Psychology, 147(1), 1-16. DOI: http://doi.org/10.1080/00223980.201 2.667016.

Brackett, M. A. y Mayer, J. D. (2003). Convergent, Discriminant, and IncrementalValidity of Competing Measures of Emotional Intelligence. Personality and Social Psychology Bulletin, 29(9), 1147-1158. DOI: http://doi.org/10.1177/0146167203254596.

Brasseur, S. y Gregoire, J. (2011). L'intelligence èmotionelle-Trait chez les adolescents á haut potential: Spédifities et liens avec la réussite scolaire et les compétences sociales. Enfance, 62(1), 59-76.

Chang, D. (2005). Self-perceived creativity, family hardiness, and emotional intelligence of Chinese giften students in Hong Kong. The Journal of Secondary Gifted Education, 16(2-3), 47-56.

Diener, E., Emmons, R., Larsen, R. J. y Griffin, S. (1985). The Satisfaction With Life Scale. Journal of Personality Assessment, 49(71-75).

Diener, E., Suh, E. M., Lucas, R. E. y Smith, H. L. (1999). Subjective well-being: Three decades of progress. Psychological Bulletin, 125(2), 276-302.

Diener, E. y Tay, L. (2015). Subjective well-being and human welfare around the world as reflected in the Gallup World Poll. International Journal of Psychology, 50(2), 135-149. DOI: http://doi.org/10.1002/ijop.12136.

Durán, A., Extremera, N. y Rey, L. (2004). Engagement and Burnout: Analysing their association patterns. Psychological Reports, 94(3), 1048-1050.

Elliot, A. J. y Thrash, T. M. (2002). Approach-avoidance motivation in personality: Approach and avoidance temperaments and goals. Journal of Personality and Social Psychology, 82, 804-818.

Esteras, J., Chorot, P. y Sandín, B. (2014). Predicción del burnout en los docentes: papel de los factores organizacionales, personales y sociodemográficos. Revista de Psicopatología Y Psicología Clínica, 19(2), 79-99.

Extremera, N., Durán, A. y Rey, L. (2007). Inteligencia emocional y su relación con los niveles de burnout, engagement y estrés en estudiantes universitarios. Revista de Educación, 342, 239-256.

Extremera, N. y Fernández-Berrocal, P. (2004). El papel de la inteligencia emocional en el alumnado: evidencias empíricas. Revista Electrónica de Investigación Educativa, 6(2). Recuperado de: http://redie.uabc.mx/redie/article/view/105.

Fredrickson, B. (2000). Cultivating positive emotions to optimize health and wellbeing. Prevention and Treatment, 3(1), 1-25.

Frijda, N., Manstead, A. S. y Bem, S. (2000). Emotions and beliefs. How feelings influence thoughts. Cambridge: Cambridge University Press. 
Garrido, M. R., Fernández, M. A., Villalba, E. B., Pérez, P. J. y Fernández, M. (2010). Evidencias de Validez de la adaptación al español de la Escala de Satisfacción con la Vida en una muestra de estudiantes universitarios. Metodología de Encuestas, 12(1), 45-62.

González, A., Danolo, D., Rinaudo, M. C. y Paoloni, P. V. (2011). Relaciones entre motivación, emoción y rendimiento académico en universitarios. Estudios de Psicología, 32(2), 257-270.

Landa, J., Lopez-Zafra, E., de Antonana, R. y Pulido, M. (2006). Perceived emotional intelligence and life satisfaction among university teachers. Psicothema, 18(1), 152-157.

Limonero, J. T., Tomás Sábado, J., Fernández-Castro, J. y Gómez-Benito, J. (2004). Influencia de la inteligencia emocional percibida en el estrés laboral de enfermería. Ansiedad y estrés, 10(1), 29-41.

Lopez-Gomez, I., Hervas, G. y Vazquez, C. (2015). An adaptation of the Positive and Negative Affect Schedules (PANAS) in a Spanish general sample. Behavioral Psychology-Psicología Conductual, 23(3), 529-548.

Lucas, R. E., Diener, C. y Larsen, R. J. (2003). Measuring positive emotions. En López, S. J. y Snyder, C. (eds.), Positive psychological assessment: a handbook of models and measures (201-218). Washington: American Psychological Association.

Lyubomirsky, S. (2001). Why are some people happier than others?: The role of cognitive and motivational processes in well-being. American Psychologist, 56, 239-249.

Lyubomirsky, S., King, L. y Diener, E. (2005). The benefits of frequent positive affect: Does happiness lead to success? Psychological Bulletin, 131, 803-855.

Nikolaou, I. y Tsaousis, I. (2002). Emotional Intelligence in the Workplace: Exploring its effects on Occupational Stress and Organisational Commitment. The International Journal of Organizational Analysis, 10(4), 327-342.

Organización para la Cooperación y el Desarrollo Económico. (2006). Attracting, Developing and Retaining Effective Teachers - Final Report: Teachers Matter.

Palomero, J. E. y Torrego, L. (2004). Europa y calidad docente ¿Convergencia o reforma educativa? Revista Interuniversitaria de Formación del Profesorado, 18(3), 23-40.

Pavot, W. y Diener, E. (2009). Review of the Satisfaction With Life Scale. Social Indicators Research Series, 39, 101-117. DOI: http://doi.org/10.1007/978-90-4812354-4 5.

Pena, M. y Repetto, E. (2008). Estado de la investigación en España sobre la inteligencia emocional en el ámbito educativo. Revista Electrónica de Investigación Psicoeducativa, 15(6), 400-420.

Pena, M., Rey, L. y Extremera, N. (2012). Life Satisfacion and engagement in Elementary and Primary Educators: differences in Emotional Intelligence and Gender. Revista de Psicodidáctica, 1(2), 341-358.

Pertegal-Felices, M. L., Castejón-Costa, J. L. y Martínez, M. Á. (2011). Competencias socioemocionales en el desarrollo profesional del maestro. Educación XX1, 14(2). DOI: http://doi.org/10.5944/educxx1.14.2.253. 
Peterson, C., Park, N. y Seligman, M. E. P. (2005). Orientations to happiness and life satisfaction: the full life versus the empty life. Journal of Happiness Studies, 6(1), 25-41. DOI: http://doi.org/10.1007/s10902-004-1278-z.

Queirós, M., Fernández-Berrocal, P., Extremera, N., Carral, J. y Queirós, P. (2005). Validación y fiabilidad de la versión portuguesa modificada de la Escala de Satisfacción Vital. Revista de Psicología, Educación Y Cultura, 9, 199-216.

Salanova, M. (2011). Introducción: Psicología Social y Psicología Positiva. Revista de Psicología Social, 26(3), 339-343.

Salovey, P., Mayer, J. D. y Caruso, D. R. (2002). The positive psychology of emotional intelligence. En R. Snyder y S. López (eds.), The handbook of positive psychology (pp. 159-171). New York: Oxford University Press.

Vázquez, C. (2009). La ciencia del bienestar. Madrid: Alianza Editorial.

Vera, J. Á., Rodríguez, C. K. y Grubits, S. (2009). La psicología social y el concepto de cultura. Psicologia \& Sociedade, 21(1), 100-107. DOI: http://doi.org/10.1590/ S0102-71822009000100012.

Vera, J., Freire, G., López, P. y García, D. (2010). Satisfacción con la Vida y Afectos Positivos en Jóvenes Universitarios. Revista Psicología e Saúde, 2, 24-41.

Vilagut, G., Ferrer, M., Rajmil, L., Rebollo, P., Permanyer-Miralda, G., Quintana, J. M., Santed, R., Valderas, J. M., Ribera, A., Domingo-Salvany, A. y Alonso, J. (2005). El Cuestionario de Salud SF-36 español: una década de experiencia y nuevos desarrollos. Gaceta Sanitaria, 19(2), 135-150.

Ware, J. E., Kosinski, M. y Keller, S. D. (1994). SF 36 physical and mental health summary scores: a user's manual. Boston: N.E.M. Center; MA: Health.

Watson, D. y Clark, L. (1984). Negative affectivity: The disposition to experience aversive emotional states. Psychological Bulletin, 96, 465-490.

Watson, D., Clark, L. y Tellegen, A. (1988). Development and validation of brief measures of positive and negative affect: The PANAS scales. Journal of Personality and Social Psychology, 54, 1063-1070.

Zijlstra, F. (2006). Stress Impact. Guildford: University of Surrey. 